\title{
HANKEL OPERATORS WITH DISCONTINUOUS SYMBOL
}

\author{
STEPHEN POWER ${ }^{1}$
}

\begin{abstract}
Douglas's localisation theory for Toeplitz operators is used to show that there exist certain Hankel operators with discontinuous symbols which do not lie in the $C^{*}$-algebra generated by the Toeplitz operators. As a simple corollary we also see that these operators do not lie in the closed linear span of the positive Hankel operators.
\end{abstract}

Introduction. Let $L^{2}$ be the Hilbert space of square integrable functions on the unit circle $T$, with complete orthonormal basis $\left\{e_{n} ; n \in Z\right\}$ where $e_{n}$ denotes the function $z^{n} . J$ is the unitary on $L^{2}$ defined by $J e_{n}=e_{-n}$ for $n$ in $Z$. $H^{2}$ denotes the Hardy subspace spanned by $\left\{e_{n} ; n \geqslant 0\right\}$ and $P$ is the orthogonal projection of $L^{2}$ onto $H^{2}$.

For an essentially bounded measurable function $\phi$ in $L^{\infty}$, the Toeplitz operator $T_{\phi}$ on $H^{2}$, is defined by $T_{\phi}=P M_{\phi} \mid H^{2}$ where $M_{\phi}$ is the usual multiplication operator on $L^{2}$. We call $\phi$ the symbol of the Toeplitz operator $T_{\phi}$. The Hankel operator on $H^{2}$, with symbol $\phi$ in $L^{\infty}$, is defined by $S_{\phi}=P J M_{\phi} \mid H^{2}$.

Whilst the Toeplitz and Hankel operators are formally rather similar, quite different techniques are usually needed to study them. However in this note we shall use Douglas's localisation theory for Toeplitz operators [2] to show that the closed linear span of the positive Hankel operators does not contain certain Hankel operators with discontinuous symbol. This result has also appeared in [4]. The proof was based on Widom's characterisation of positive Hankel operators [5] which we avoid here.

Notation. For $\phi$ in $L^{\infty}$, the functions $\phi^{*}$ and $\tilde{\phi}$ are defined by

$$
\phi^{*}(z)=\phi(\bar{z}) \text { and } \tilde{\phi}(z)=\overline{\phi^{*}(z)} \quad(z \in T) .
$$

Thus we have $J M_{\phi}=M_{\phi} . J$.

Let $\mathbf{T}$ denote the $C^{*}$-algebra generated by the Toeplitz operators and let $\mathbf{P}$ denote the closed linear span of the positive Hankel operators.

The following simple lemma shows that the symbol of the adjoint of a

Received by the editors November $8,1976$.

AMS (MOS) subject classifications (1970). Primary 47B35, 47C10.

Key words and phrases. Hankel operator, discontinuous symbol, Toeplitz operator, $C^{*}$-algebra, positive Hankel operator.

${ }^{1}$ This research was supported by the Science Research Council.

- American Mathematical Society 1977 
Hankel operator is not the 'local adjoint' of its symbol. The proof of Theorem 8 rests on this fact.

LEMMA 1. $S_{\phi}^{*}=S_{\tilde{\phi}}$ for $\phi \in L^{\infty}$.

Proof. For $f$ and $g$ in $H^{2}$ we have

$$
\left(S_{\phi} f, g\right)=\left(J M_{\phi} f, g\right)=\left(M_{\phi^{*}} J f, g\right)=\left(J f, M_{\tilde{\phi}} g\right)=\left(f, S_{\tilde{\phi}} g\right) .
$$

LEMMA 2. $S_{\phi}^{*} S_{\psi}=T_{\bar{\phi} \psi}-T_{z \bar{\phi}} T_{\bar{z} \psi}$ for $\phi, \psi \in L^{\infty}$.

Proof.

$$
S_{\phi}^{*} S_{\psi}=P J M_{\tilde{\phi}} P J M_{\psi}\left|H^{2}=P M_{\phi}^{-} J P J M_{\psi}\right| H^{2}=P M_{\bar{\phi} z}\left(M_{\bar{z}} J P J M_{z}\right) M_{\bar{z} \psi} \mid H^{2} .
$$

Since $M_{z} J P J M_{z}=I-P$ we have

$$
S_{\phi}^{*} S_{\psi}=P M_{\bar{\phi} z}(I-P) M_{\bar{z} \psi} \mid H^{2}=T_{\bar{\phi} \psi}-T_{z \bar{\phi}} T_{\bar{z} \psi} \text {. }
$$

\section{LEMMA 3. $\mathbf{P} \subset \mathbf{T}$.}

Proof. Let $S$ be a positive Hankel operator. Lemma 2 shows that $S^{2} \in \mathrm{T}$. Since $S$ is the unique positive square root of $S^{2}$ and $\mathrm{T}$ is a $C^{*}$-algebra we have $S \in \mathbf{T}$ and the lemma follows.

A well-known classical theorem for Hankel operators is due to Hartman (see [3] or [1]). This states that a Hankel operator is compact if and only if its symbol can be chosen to be continuous. Since $S_{\phi}=0$ only when $\phi \in z H^{\infty}$ it follows that $S_{\phi}$ is compact if and only if $\phi \in H^{\infty}+C(T)$. We shall use this result in Theorem 8 .

LEMMA 4. If $\phi, \psi \in L^{\infty}$ and if $\phi$ or $\psi$ is continuous then $T_{\phi \psi}-T_{\phi} T_{\psi}$ is compact.

Proof. Lemma 2 shows that $S_{z \bar{\phi}}^{*} S_{z \psi}=T_{\phi \psi}-T_{\phi} T_{\psi}$ and Hartman's theorem completes the proof. Alternatively see [2, p. 184].

The following two localisation theorems appear in Douglas [2, pp. 198, 199].

For $\lambda \in T$, let $J_{\lambda}$ be the closed ideal in T generated by $\left\{T_{\phi}: \phi \in C(T), \phi(\lambda)\right.$ $=0\}$ and let $\Phi_{\lambda}$ be the natural *-homomorphism of $\mathbf{T}$ onto the quotient $C^{*}$. algebra $\mathbf{T}_{\lambda}=\mathbf{T} / J_{\lambda}$. Let $\Phi$ be the *-homomorphism from $\mathbf{T}$ to $\Sigma_{\lambda \in T} \oplus \mathbf{T}_{\lambda}$ defined by $\Phi=\sum_{\lambda \in T} \oplus \Phi_{\lambda}$.

THEOREM 5. ker $\Phi$ contains the ideal of compact operators $K$ and $\Phi$ induces $a$ *-isometrical isomorphism

$$
\Phi_{c}: \mathbf{T} / K \rightarrow \sum_{\lambda \in T} \oplus \mathbf{T}_{\lambda}
$$

Let $\Lambda$ be the maximal ideal space of $L^{\infty}$ and let $F_{\lambda}=\{m \in \Lambda ; m(z)=\lambda\}$ be the fibre in $\Lambda$ over $\lambda$ for $\lambda \in T$. 
THEOREM 6. If $\left\{\phi_{i j}\right\}_{i, j=1}^{n}$ are functions in $L^{\infty}$ with Gelfand transforms $\hat{\phi}_{i j}$ on $\Lambda$ and $\lambda \in T$ then $\Phi_{\lambda}\left(\Sigma_{i} \Pi_{j} T_{\phi_{i j}}\right)$ depends only on the functions $\left\{\hat{\phi}_{i j} \mid F_{\lambda}\right\}_{i, j=1}^{n}$.

Corollary 7. If $\phi, \psi \in L^{\infty}$ and $\psi$ is continuous on a neighborhood of $\lambda \in T$ then $\Phi_{\lambda}\left(T_{\phi \psi}-T_{\phi} T_{\psi}\right)=0$.

Proof. Theorem 6 implies that we can assume $\psi$ to be continuous on $T$. The corollary then follows from Lemma 4 and Theorem 5.

THEOREM 8. Let $\alpha$ be a nonreal complex number of modulus one and let $\phi$ be a function in $L^{\infty}$ which is continuous on $T$ apart from a (proper) jump discontinuity at $\alpha$. Then $S_{\phi} \notin \mathbf{T}$.

Proof. By Lemma 2, $S_{\phi}^{*} S_{\phi}=T_{|\phi|^{2}}-T_{z \phi} T_{\bar{z} \phi}$ and so by Corollary 7 $\Phi_{\lambda}\left(S_{\phi}^{*} S_{\phi}\right)=0$ for $\lambda \neq \alpha$.

Suppose now that $S_{\phi} \in \mathrm{T}$. Since $\Phi_{\lambda}$ is a ${ }^{*}$-homomorphism $\Phi_{\lambda}\left(S_{\phi}\right)^{*} \Phi_{\lambda}\left(S_{\phi}\right)$ $=0$ for $\lambda \neq \alpha$. Similarly, since by Lemma $1 S_{\phi}^{*}=S_{\tilde{\phi}}$ and $\tilde{\phi}$ is continuous apart from a jump discontinuity at $\bar{\alpha}$, it follows that $\Phi_{\lambda}\left(S_{\phi}\right) \Phi_{\lambda}\left(S_{\phi}\right)^{*}=0$ for $\lambda \neq \bar{\alpha}$. Thus $\Phi_{\lambda}\left(S_{\phi}\right)=0$ for all $\lambda \in T$. By Theorem $5 S_{\phi}$ is compact and so by Hartman's theorem $\phi \in H^{\infty}+C(T)$. Since such functions cannot have jump discontinuities we conclude that $S_{\phi} \notin \mathbf{T}$.

COROLlARY 9. Let $\phi$ be a function in $L^{\infty}$ which is continuous apart from a jump discontinuity at a nonreal point of the unit circle. Then $S_{\phi}$ does not lie in $\mathbf{P}$.

Proof. Immediate from Theorem 8 and Lemma 3.

The corollary does not remain true if the discontinuities occur at -1 or +1 . In fact in [4] it is shown that if $\phi$ is continuous apart from jump discontinuities at +1 or -1 then $S_{\phi} \in \mathbf{P}$.

It would be interesting to settle whether $T$ contains any other Hankel operators besides those in $\mathbf{P}$.

\section{REFERENCES}

1. F. F. Bonsall and S. C. Power, A proof of Hartman's theorem on compact Hankel operators, Math. Proc. Cambridge Philos. Soc. 78 (1975), 447-450.

2. R. G. Douglas, Banach algebra techniques in operator theory, Academic Press, New York, 1973.

3. P. Hartman, On completely continuous Hankel matrices, Proc. Amer. Math. Soc. 9 (1958), 862-866.

4. S. C. Power, Intertwining operators, Ph.D. Thesis, Univ. of Edinburgh, 1976.

5. H. Widom, Hankel matrices, Trans. Amer. Math. Soc. 121 (1966), 1-35.

Department of Mathematics, University of Edinburgh, Edinburgh, SCotland

Current address: Department of Mathematics, Dalhousie University, Halifax, Nova Scotia B3H 3J5, Canada 